\title{
ACR
}

Selected Papers of \#AolR2020:

The 21 ${ }^{\text {st }}$ Annual Conference of the

Association of Internet Researchers

Virtual Event / 27-31 October 2020

\section{DETECTION ALGORITHMS AND THEIR IMPLICATIONS FOR PARTICIPATION: THE CASE OF MASHUPS}

\author{
Ragnhild Brøvig-Hanssen \\ University of Oslo \\ Ellis Jones \\ University of Oslo
}

Mashup music has often been seen as an exemplar of the participatory cultural environment that many expected the internet to foster. However, many online platforms today utilise automated and algorithmically driven tools to detect and moderate unwelcome content, including that which is seen as copyright infringing. Drawing on interviews and an online survey with mashup producers, we show that detection procedures have a strong negative impact on mashup producers, including on their overall motivation to create mashups. We argue that that platforms' technological (and economic) power currently supersedes, in practice, the legal exceptions intended to permit particular copyright uses. Thus, we contribute to a growing literature highlighting the consequences of the immense power held by platforms to monitor, amend and remove content, and the central role of automatic and algorithmic regulatory tools in enacting this power.

Mashup music is a form of remix that relies on the combining of pre-existing, recognisable recordings. Since these samples are usually unauthorised, it holds an ambiguous legal status. The copying of an artwork without authorisation from its copyright holder(s) generally constitutes copyright infringement. Yet, various countries' copyright laws are softened by exceptions, including 'fair use' (in the US), and parody (in the EU, among others), which are intended to balance the rights of intellectual property with fundamental rights of freedom of expression. Several scholars have argued that many examples of creative and transformative uses of samples in fact

Suggested Citation (APA): Brøvig-Hanssen, Ragnhild and Jones, Ellis. (2020, October). Detection Algorithms and their Implcations for Participation: The Case of Mashups. Paper presented at AolR 2020: The $21^{\text {th }}$ Annual Conference of the Association of Internet Researchers. Virtual Event: AolR. Retrieved from http://spir.aoir.org. 
correspond to specific exceptions, and that corporate interests have taken advantage of legal vagueness in ways that damage culturally valuable art expressions.

At the turn of the twenty-first century, when mashup emerged, the internet was seen to offer a means by which to bypass these strong copyright restrictions and circulate sample-based music outside of traditional cultural industries' infrastructures. However, following economic and legal pressure from copyright holders and media corporations, many platforms have now implemented robust detection procedures which include the use of automated identification tools to prevent the circulation of purportedly copyrightinfringing content. Upon detection, these tools offer copyright holders different options, including to ignore the use, to monetise it by running ads against it and collecting the revenue, or to block it altogether.

We interviewed thirty mashup producers in 2019 , with the aim of understanding the impact of these detection tools on the mashup scene. The same year, we also surveyed ninety-two mashup producers via an anonymous online form. This empirical data shows that engagements with platforms' detection procedures are an 'everyday' reality for mashup producers. $96 \%$ of survey respondents had experienced the detection of copyrighted material in their mashups by platforms. $82 \%$ had experienced the removal of their mashups from platforms, and $53 \%$ of them had had accounts permanently deleted as a direct consequence of uploading mashups. Although there is usually some recourse to appeal takedowns on platforms, only $40 \%$ of survey respondents with takedown experience had ever explored this option. This reticence to appeal is often due to fear that this might draw further attention to their account and therefore result in more severe consequences, such as account deletion. We explore the impacts of these experiences in the following three areas: on motivation, distribution, and creative choices.

Our survey clearly shows that mashup producers see platforms' detection procedures as a hindrance to their activity. Of the surveyed producers, $56 \%$ reported that platforms' detection procedures made them less motivated, compared to just $2 \%$ who felt that such procedures made them more motivated. A key source of frustration is that what is lost after a takedown is not only the music but also the play counts and, in the case of account deletion, the follower count and access to those followers. These losses can represent a really significant, demotivating experience which can happen at any timeeven mashups which have been online for years can disappear 'overnight'.

Our survey results also indicate that detection procedures have a significant impact on where producers distribute their mashups (with $81 \%$ answering that such procedures impact their distribution choices either 'somewhat' or 'to a great extent'). Interviewees indicated SoundCloud, YouTube, Facebook, Twitter, and Instagram as platforms whose detection procedures made them 'unsafe' for mashups. YouTube was seen as particularly prone to takedowns, but its uniquely large audience meant that it was still considered worth the effort to try and upload mashups there. The market-leading music streaming platforms, Spotify and Apple Music, were generally seen to be infeasible due to their especially strict copyright regulation. 
Furthermore, producers often employed distribution tactics aimed at minimising their potential losses. This included the use of 'test' accounts, where producers will upload new material on a different account in order to see how the platforms' algorithm reacts, thus reducing the risk of their 'main account' being hit with copyright strikes. Another mitigating tactic is the use of alternative platforms, developed more or less specifically for mashup producers. These sites have similar features to well-known platforms and offer a much higher degree of 'safety' to individual mashup producers, but at the cost of a much reduced audience.

Detection procedures also affected mashup producers' creative decisions, since many of the small-scale tactics used to bypass detection involve changing the content of the mashup music or video itself. The 'masking' techniques they use to keep their music clear of the automated detection tools include 'pitch shifting', speed alteration, added processing effects, or altered videos, all beyond the extent required simply to make the sampled sources fit together. Yet, producers are often unwilling to compromise on this aspect within a form which relies heavily on the recognisability of the original samples. Our data also shows that detection procedures impact the creative process in terms of the music that producers choose to sample in the first place; certain artists' recordings are widely perceived as 'off limits' for use in mashups because of their high propensity to result in takedowns. The aesthetic restrictions imposed by detection procedures can also lead to motivational issues.

The legal status of mashup music is complex, but there are certain indications that mashups are not definitively excluded from being considered legal, by virtue of copyright exceptions. So, while platforms might act to remove or alter material on the grounds of its presumed illegality, the legal status of mashups is in fact still an open question. In the meantime, the practical reality is that mashups (and the larger remix culture of which they are a part) face a threat that borders on the existential. Any rapid solution to this state of affairs seems unlikely: automated detection tools are a long way from boasting the necessary flexibility to make nuanced judgements, and non-automatic tools cannot be expected to manage the scale of content involved. Mashup music, and remix culture more generally, is in this regard just one place among many where the current economic and technological power of online platforms has brought significant effects to bear on cultural producers-effects which are far from what was anticipated at the dawn of Web 2.0.1

\footnotetext{
${ }^{1}$ Please feel free to contact the authors for a full-version of this paper. This work is supported by the University of Oslo and the Research Council of Norway through its Centres of Excellence scheme (RITMO, 262762) and research project MASHED (275441) led by R. Brøvig-Hanssen.
} 